\title{
Mahler's Conjecture and Wavelets
}

\author{
K. Ball \\ Department of Mathematics, University College London, \\ Gower Street, London WC1E 6BT, England \\ kmb@math.ucl.ac.uk
}

\begin{abstract}
It is shown that a special case of Mahler's conjecture can be reformulated in terms of the solutions to the scaling equation of wavelet theory.
\end{abstract}

\section{Introduction}

This paper describes a connection between the well-known geometric problem of Mahler concerning the volumes of convex bodies and their polars, and the theory of wavelets which has attracted so much recent attention. A special case of Mahler's conjecture leads naturally to a question about solutions of the scaling equation which appears in the construction of wavelets. This latter question is not completely natural for wavelet theorists because the inequality "goes the wrong way". However, a solution of this question would shed considerable light on the rather mysterious behaviour of the scaling equation and one may hope that some insight can be gained from a consideration of the underlying geometric problem.

The paper is in three parts. The first describes the special case of Mahler's conjecture which is to be considered and expresses this geometric problem in probabilistic form. The second part explains the appearance of the scaling equation and its geometric significance. The third part contains a final statement of the problem and discusses several situations in which the proposed inequality is sharp.

\section{A Special Case of Mahler's Conjecture}

Let $K$ be a symmetric convex body in $\mathbb{R}^{n}$, and let $K^{*}$ be its polar $\{x:|\langle y, x\rangle| \leq 1$ for all $y \in K$ \}. Mahler's conjecture asserts that

$$
\operatorname{vol}(K) \cdot \operatorname{vol}\left(K^{*}\right) \geq \frac{4^{n}}{n !},
$$


there being equality for the cube/octahedron pair (in each dimension) and for other pairs of bodies. Mahler originally made this conjecture in connection with the successive minima of convex bodies which appear in the geometry of numbers. The strongest result to date, in the direction of Mahler's conjecture, is the theorem of Bourgain and Milman [BM] which shows that the inequality holds up to a factor of (constant) $)^{n}$, the constant being independent of everything.

The expression $\operatorname{vol}(K) \operatorname{vol}\left(K^{*}\right)$ is an affine invariant of $K$ and so there is equality in (1) whenever $K$ is a parallelepiped -i.e., $K$ has $2 n$ facets. A sharp upper estimate for this invariant is provided by the Blaschke-Santalo inequality

$$
\operatorname{vol}(K) \cdot \operatorname{vol}\left(K^{*}\right) \leq\left(v_{n}\right)^{2},
$$

$v_{n}$ being the volume of the $n$-dimensional Euclidean unit ball,

$$
v_{n}=\frac{\pi^{n / 2}}{\Gamma(n / 2+1)}
$$

Since $v_{n}^{2}$ is roughly $(2 \pi e / n)^{n}$, the ratio of the upper and conjectured lower bounds is of the order of (constant) ${ }^{n}$.

Since Mahler's conjecture says nothing for symmetric bodies with $2 n$ facets it is reasonable to ask what happens if $K$ has $2 n+2$ facets. Such a $K$ can be realized, up to affine invariance, as a one-codimensional section (through the centre) of an $(n+1)$-dimensional cube. If $H$ is an $n$-dimensional subspace of $\mathbb{R}^{n+1}$ and

$$
K=H \cap[-1,1]^{n+1},
$$

then the polar of $K$ in $H$ is the orthogonal projection of the "octahedron"

$$
B_{1}^{n+1}=\left\{x: \sum\left|x_{i}\right| \leq 1\right\}
$$

onto $H$. Replacing $n+1$ by $n$, the special case of Mahler's conjecture, for bodies with one extra pair of facets, can be stated as follows:

If $H$ is a one-codimensional subspace of $\mathbb{R}^{n}$ and $P$ is the orthogonal projection onto $H$, then

$$
\operatorname{vol}\left(H \cap[-1,1]^{n}\right) \cdot \operatorname{vol}\left(P\left(B_{1}^{n}\right)\right) \geq \frac{4^{n-1}}{(n-1) !}
$$

If $Q_{n}$ is taken to be the unit cube $\left[-\frac{1}{2}, \frac{1}{2}\right]^{n}$, the inequality rescales as

$$
\operatorname{vol}\left(H \cap Q_{n}\right) \cdot \operatorname{vol}\left(P\left(B_{1}^{n}\right)\right) \geq \frac{2^{n-1}}{(n-1) !}
$$


The volume of $P\left(B_{1}^{n}\right)$ is easy to express in terms of the unit normal $a=\left(a_{i}\right)_{1}^{n}$ to $H$. The projection onto $H$ of the surface of $B_{1}^{n}$ covers almost all of $P\left(B_{1}^{n}\right)$ exactly twice. The surface is composed of $2^{n}$ facets indexed by the choices of $n$ signs $\varepsilon=\left(\varepsilon_{i}\right)_{1}^{n}$. Each facet has $(n-1)$-dimensional volume $\sqrt{n} /(n-1)$ ! and the volume of the projection of the $\varepsilon$-facet is

$$
\frac{\sqrt{n}}{(n-1) !} \cdot \frac{1}{\sqrt{n}}|\langle a, \varepsilon\rangle|
$$

since $(1 / \sqrt{n}) \varepsilon$ is the unit normal to the $\varepsilon$-facet. Thus

$$
\begin{aligned}
\operatorname{vol}\left(P\left(B_{1}^{n}\right)\right) & =\frac{1}{2} \sum_{\varepsilon} \frac{1}{(n-1) !}|\langle a, \varepsilon\rangle| \\
& =\frac{2^{n-1}}{(n-1) !} \cdot \frac{1}{2^{n}} \sum_{\varepsilon}|\langle a, \varepsilon\rangle| \\
& =\frac{2^{n-1}}{(n-1) !} E\left|\sum_{1}^{n} a_{i} U_{i}\right|,
\end{aligned}
$$

where $U_{1}, \ldots, U_{n}$ are iid random variables with $P\left(U_{i}=1\right)=P\left(U_{i}=-1\right)=\frac{1}{2}$. Mahler's conjecture for bodies with two extra facets is thus

$$
\operatorname{vol}\left(H \cap Q_{n}\right) \cdot E\left|\sum_{1}^{n} a_{i} U_{i}\right| \geq 1
$$

for all unit vectors $a$ and $H=\langle a\rangle^{\perp}$.

The Khintchine inequality states that, for some constant $C$,

$$
\left(\sum_{1}^{n} a_{i}^{2}\right)^{1 / 2} \leq C E\left|\sum_{1}^{n} a_{i} U_{i}\right|
$$

for any sequence $\left(a_{i}\right)_{1}^{n}$ (and all $n$ ). It was conjectured by Littlewood that the sharp constant here is $C=\sqrt{2}$. This was proved by Szarek [S]. On the other hand, it was shown by the author [B] that, for any $K$, $\operatorname{vol}\left(H \cap Q_{n}\right) \leq \sqrt{2}$. Thus Szarek's result would follow from the cube-slicing theorem together with (4).

The quantity $\operatorname{vol}\left(H \cap Q_{n}\right)$ can also be expressed using the iid random variables $\left(U_{i}\right)_{1}^{n}$. This is perhaps most easily seen by evaluating the integrals in [B] using residue calculus. The result is that if $a$ is a unit vector with all $a_{i}$ nonzero and $H=\{a\}^{\perp}$, then

$$
\operatorname{vol}\left(H \cap Q_{n}\right)=\frac{1}{(n-1) ! \prod_{1}^{n} a_{i}} E\left[\left(\Pi U_{j}\right) \operatorname{sgn}\left(\sum a_{j} U_{j}\right) \cdot\left(\sum a_{j} U_{j}\right)^{n-1}\right] .
$$


Inequality (4) thus states that, for any sequence $\left(a_{i}\right)_{1}^{n}$,

$$
(n-1) ! \prod a_{i} \leq E\left[\left(\prod U_{j}\right) \operatorname{sgn}\left(\sum a_{j} U_{j}\right)\left(\sum a_{j} U_{j}\right)^{n-1}\right] E\left|\sum a_{i} U_{i}\right|
$$

This form of the inequality is made highly suggestive by noting that

$$
n ! \prod a_{i}=E\left[\left(\prod U_{j}\right)\left(\sum a_{j} U_{j}\right)^{n}\right]
$$

Nevertheless, inequality (5) does not seem to be easy to attack. The approach described below looks more promising.

In view of the probabilistic form of $\operatorname{vol}\left(P\left(B_{1}^{n}\right)\right)$ it is natural to ask for a simple probabilistic expression for $\operatorname{vol}\left(H \cap Q_{n}\right)$. Such a representation was already used in [B] and [H]. If $Z_{1}, \ldots, Z_{n}$ are iid random variables, uniformly distributed on $\left[-\frac{1}{2}, \frac{1}{2}\right]$, then the random vector $\left(Z_{1}, \ldots, Z_{n}\right)$ induces Lebesgue measure on $Q_{n}$. If, for each $r \in \mathbb{R}$,

$$
\varphi(r)=\operatorname{vol}\left((H+r a) \cap Q_{n}\right)
$$

so that $\varphi$ is the function obtained by scanned $Q_{n}$ with translates of $H$, then it is easy to see that $\varphi$ is the density of the random variable $\sum_{1}^{n} a_{i} Z_{i}$. For reasons that will become apparent, it is convenient to replace the random variables $\left(U_{i}\right)$ by another sequence, half as large. If $\left(V_{i}\right)_{1}^{n}$ are iid with $P\left(V_{i}=\frac{1}{2}\right)=P\left(V_{i}=-\frac{1}{2}\right)=\frac{1}{2}$, then inequality (4) states that, with $\varphi$ as above,

$$
\varphi(0) E\left|\sum a_{i} V_{i}\right| \geq \frac{1}{2}
$$

\section{The Appearance of the Scaling Equation}

The similar forms of the random variables

$$
X=\sum a_{i} V_{i} \text { and } Y=\sum a_{i} Z_{i}
$$

which (effectively) appear in (6) makes it possible to express the relationship between $X$ and $Y$ without reference to their origins as linear combinations of other random variables. If $\left(V_{j}\right)_{1}^{\infty}$ is an iid sequence with each $V_{j}$ distributed equally on the points $\pm \frac{1}{2}$, then the random variable

$$
\sum_{1}^{\infty} 2^{-j} V_{j}
$$


is uniformly distributed on the interval $\left[-\frac{1}{2}, \frac{1}{2}\right]$. Hence if $\left(X_{j}\right)_{1}^{\infty}$ is an iid sequence of copies of $X$,

$$
\sum_{1}^{\infty} 2^{-j} X_{j}
$$

is distributed like $Y$. Inequality (6) thus states that

$$
\varphi(0) E|X| \geq \frac{1}{2}
$$

if $\varphi$ is the density of $Y=\sum_{1}^{\infty} 2^{-j} X_{j}$. Inequality (7) is probably too much to expect for a general symmetric random variable $X$ (as opposed to the special ones discussed above). However, (7) can be weakened without any information being lost in the special case. If $\varphi$ is obtained by scanning a cube, then it attains its maximum value at 0 : this follows from the Brunn-Minkowski inequality among other things. So for the purpose of establishing Mahler's conjecture for one-codimensional sections of the cube it would suffice to obtain

$$
\|\varphi\|_{\infty} E|X| \geq \frac{1}{2}
$$

for $\varphi$ the density of $\sum_{1}^{\infty} 2^{-j} X_{j}$.

The relationship

$$
Y=\sum_{1}^{\infty} 2^{-j} X_{j}
$$

is already recognizable to wavelet theorists. If $X$ and $\left(X_{j}\right)_{1}^{\infty}$ are idd, then $X+Y$ has the same distribution as $2 Y$ so that if $Y$ has density $\varphi$, and $P$ is the law of $X$, then

$$
\varphi(y)=2 \int_{\mathbb{R}} \varphi(2 y-x) d P(x)
$$

This equation is the so-called two-scale relation for the functions $\varphi$ used in the construction of wavelets. (The actual wavelet, $W$, associated to $\varphi$ is given by $W(y)=2 \varphi(2 y)-\varphi(y)$.) If $X$ is a random variable of the form $\sum a_{i} V_{i}$, then (9) has a simple geometric interpretation which depends upon the decomposition of a cube into $2^{n}$ cubes of half the size. For example, when $y=0$, (9) says that the volume of the central slice of $H \cap Q_{n}$ is twice the average of the volumes of the parallel slices through the corners of $Q_{n}$ : equivalently, that it is $1 / 2^{n-1}$ times the sum of the volumes of these parallel slices. This is immediately apparent from Fig. 1.

\section{The Problem}

The upshot of the foregoing discussion is the following conjecture concerning the scaling functions $\varphi$ which appear in the construction of wavelets. Suppose $P$ is the 

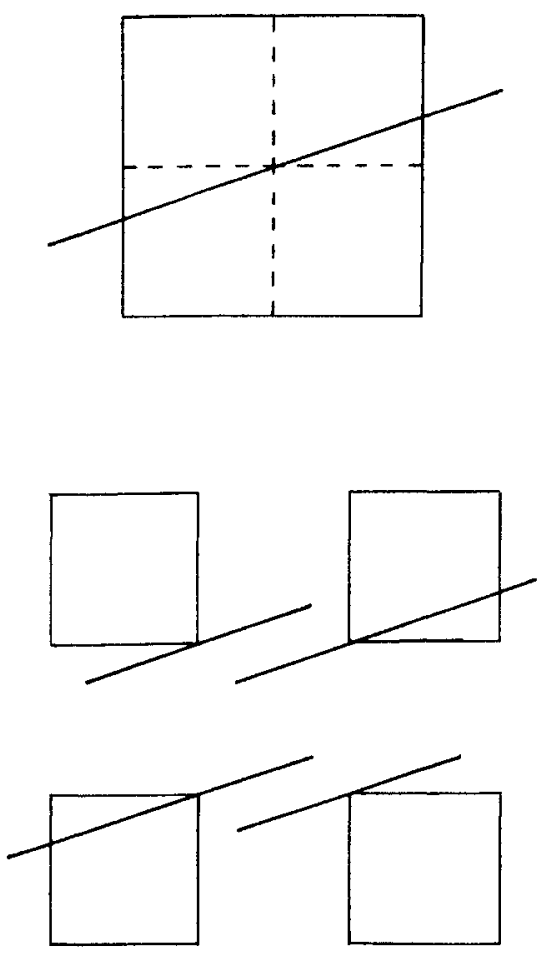

Fig. 1

law of a symmetric random variable $X$ and there is a nonnegative integrable $\varphi$ satisfying $\int \varphi=1$ and

$$
\varphi(y)=2 \int_{-\infty}^{\infty} \varphi(2 y-x) d P(x), \quad y \in \mathbb{R}
$$

then

$$
\|\varphi\|_{\infty} E|X| \geq \frac{1}{2}
$$

(The existence of $\|\varphi\|_{\infty}$ is not really a necessary hypothesis if $\|\varphi\|_{\infty}$ is understood to be infinite when no bounded $\varphi$ exists.) As mentioned in the Introduction, inequality (10) goes the "wrong way" for applications to wavelets: it says that $\varphi$ cannot be too well behaved. On the other hand, any possibility of a sharp inequality involving solutions of the scaling equation, looks intriguing. It should be remarked that if the inequality is relaxed sufficiently, it ceases to be a problem. $\|\varphi\|_{\infty} E|X| \geq \frac{1}{4}$ is trivial, as is the sharp estimate $\|\varphi\|_{\infty}\left(E X^{2}\right)^{1 / 2} \geq \frac{1}{2}$. 
Finally, it is worth noting that if (10) is true, it is exact for a wide variety of random variables $X$. If $\left(V_{i}\right)_{1}^{\infty}$ is an iid $\pm \frac{1}{2}$ sequence,

$$
X=\sum_{1}^{\infty} a_{i} V_{i}
$$

and $a_{1} \geq \sum_{2}^{\infty}\left|a_{i}\right|$, then the "slice of the infinite cube" perpendicular to $\left(a_{i}\right)_{1}^{\infty}$ is a cube: hence, there is equality in Mahler's conjecture. An amusing special case occurs when $X$ is uniformly distributed on $\left[-\frac{1}{2}, \frac{1}{2}\right]$ (or any other symmetric interval). This corresponds to the choice $a_{i}=2^{-i}$ in (11). In this case $\varphi$ is supported on $\left[-\frac{1}{2}, \frac{1}{2}\right]$ and one easily gets from the scaling equation

$$
\varphi(0)=2 \int_{-1 / 2}^{-1 / 2} \varphi(x) d x=2
$$

$\left(E|V|=\frac{1}{4}\right.$ in this case.)

There is a further case of equality which is isolated and does not fit the above pattern. The slices of a four-dimensional cube, which are perpendicular to its diagonals, are three-dimensional regular octahedra and so satisfy Mahler's conjecture with equality. The associated spline $\varphi$ is the basic cardinal cubic spline: the nonzero cubic spline with equally spaced knots whose support is shortest. It is my feeling that this pathological case of equality is the major barrier to a proof of (10).

Standard references for wavelets are [C] and [D].

\section{References}

[B] K. M. Ball, Cube slicing in $\mathbb{R}^{n}$, Proc. Amer. Math. Soc. 97 (1986), 465-473.

[BM] J. Bourgain and V. D. Milman, New volume ratio properties for convex symmetric bodies in $\mathbb{R}^{n}$, Invent. Math. 88 (1987), 319-340.

[C] C. K. Chui, An Introduction to Wavelets, Academic Press, New York, 1992.

[D] 1. Daubechies, Ten Lectures on Wavelets, CBMS-NSF Regional Conference Series in Applied Mathematics, SIAM, Philadelphia, PA, 1992.

[H] D. Hensley, Slicing the cube in $\mathbb{R}^{n}$ and probability, Proc. Amer. Math. Soc. 73 (1979), 95-100.

[S] S. J. Szarek, On the best constant in the Khinchine inequality, Studia Math. 58 (1976), 197-208. 